\title{
Prediction of Interaction Sites of PcRab7-VP28
}

\author{
Xiaofeng Ji, Yuan Zheng, Jun Sheng* \\ Yellow Sea Fisheries Research Institute, Chinese Academy of Fishery Sciences, Qingdao, China \\ Email: *qdjixf@163.com
}

How to cite this paper: Ji, X.F., Zheng, Y. and Sheng, J. (2021) Prediction of Interaction Sites of PcRab7-VP28. Computational Molecular Bioscience, 11, 50-56. https://doi.org/10.4236/cmb.2021.112003

Received: April 26, 2021

Accepted: May 16, 2021

Published: May 19, 2021

Copyright $\odot 2021$ by author(s) and Scientific Research Publishing Inc. This work is licensed under the Creative Commons Attribution International License (CC BY 4.0).

http://creativecommons.org/licenses/by/4.0/

\section{(c) (i) Open Access}

\begin{abstract}
White spot syndrome virus (WSSV) is one of the most important pathogens in the world. Since its outbreak in 1993, the virus has caused huge economic losses. Studies have confirmed that in the early stage of infection, VP28, the main envelope protein of WSSV, as a viral adhesion protein, binds to PcRab7 of Penaeus chinensis to help the virus enter the host cells. Understanding the mechanism of PcRab7-VP28 interaction is of great significance to understand the mechanism of WSSV infection and the development of antiviral drugs. In this research, the interaction interface and interaction sites were predicted by using the methods of molecular simulations. Results showed that VP28 binds to the second $\beta$-sheet (L73-D86) of PcRab7, which is consistent with the region detected in previous studies. Furthermore, we speculated the possible interaction sites in PcRab7 are E81, F77 and D76. These results may contribute to a deep understanding of the infection mechanism of WSSV on the host.
\end{abstract}

\section{Keywords}

White Spot Syndrome Virus (WSSV), Interaction Sites, Penaeus

\section{Introduction}

White spot syndrome is a kind of syndrome caused by white spot syndrome virus (WSSV). It is one of the main diseases of shrimp epidemic outbreak, with high mortality and serious harm to shrimp breeding industry. It is an important aquatic animal infectious disease to be reported by OIE [1]. Since the outbreak of the disease in 1992, it caused a large area of death of prawns, and has still brought great economic losses to China's prawn breeding industry. The prevention and treatment of white spot syndrome is of great significance to the healthy cultivation of shrimp in China [2].

WSSV is the first marine biological DNA virus to obtain the whole genome in the world. The genome size of WSSV is about $300 \mathrm{~KB}$. It is predicted that WSSV 
encodes about 180 proteins, among which more than 50 proteins encoded by WSSV virus have been identified as structural proteins [3]. Among these structural proteins, envelope protein is an important determinant of viral infection and pathogenicity [4], which is considered to be the first molecule in direct contact with the host [5] [6], so it plays a key role in cell targeting and host defense. Although the exact mechanism of WSSV virus entry into shrimp is still unknown, it is speculated that the virus entry into host cells is through the interaction between virus envelope protein and host cell receptor [7]. Since the virus enters the host cell through the interaction between the virus envelope protein and the host cell receptor, it is very important for the discovery of interaction blockers, especially for the development of antiviral drugs [8].

Among all of the known structural proteins of WSSV, VP28 is the main envelope [9] protein, encoded by the reading frame wsv421 [10]. The crystal structure of VP28 showed that VP28 is a trimmer with triple symmetry. Confirmed by immune-electron microscopy, VP2 8 was distributed on the outer surface of the virus [11]. In the process of infection, VP28, as a virus binding protein, binds to Rab7 protein in prawn cells and mediates the fusion of virus envelope and host cell membrane, thus helping the virus enter the host cell [12] [13], which plays a key role in the early stage of WSSV infection of prawn [14]. In the study of WSSV molecular mechanism, it was found that Rab7 (PmRab7) protein in Penaeus monodon and PcRab7 in Penaeus chinensis can be specifically combined with VP28. However, the interaction mechanism of PcRab7 and VP28 remains unknown.

Although analysis of the crystal tertiary structure of protein complex is the most direct way to clarify the interaction mechanism, due to the limitation of experimental conditions the crystal tertiary structure of Rab7 and VP28 has not been obtained. The molecular mechanism of the interaction has not been fully elucidated. The development of structural modeling and molecular simulation technology and the improvement of simulation accuracy provide a new way and method for interaction research. In this research, we determined the interaction interface and interaction sites by using the methods of molecular simulation. This study will contribute to a deep understanding of the infection mechanism of WSSV on the host.

\section{Methods}

The target sequence of PcRab7 (accession NO. AEF33797) was downloaded from protein database NCBI. Meanwhile, a sequence alignment for PcRab7 against other sequences with available tertiary structure was performed on the online server BLAST (http://blast.ncbi.nlm.nih.gov). The automated sequence alignment and analysis of the template and target was carried out using the program of Espript2.2 [15]. The tertiary structure of PcRab7 was built by I-TASSER [16], which is a hierarchical approach to protein structure prediction. The predicted structures were saved in the PDB format. Furthermore, we select PROCHECK 
[17] to evaluate the quality of the generated models. Select PcRab7 and VP28 as the single structure and docked by employed the protein-protein docking program ZDOCK3.0.2 [18]. After docking, 2000 structures of PcRab7-VP28 complex were generated and sorted according to binding energy, the complex with the lowest binding free energy was as the initial for further analysis.

\section{Results and Discussion}

Through BLAST template search based on the protein data bank (PDB), it can be seen that the sequence consistency of PcRab7 and four Rab7 proteins with known crystal tertiary structure is between $86 \%-91 \%$, and the sequence coincidence rate is more than 78\% (Figure 1). The differences between PcRab7 and the other four homologous proteins are mainly concentrated in the two ends of the protein, which indicates that the structure constructed with these four homologous structures as templates is reliable.

Using 1VG0 as the template, the three-level structure of PcRab7 was constructed (Figure 2(A)). According to the Ramachandran plot test (Figure 2(B)), 98.75\% of the regions in the structure are within the acceptable range.

The constructed structure of PcRab7 was used for molecular docking with protein-protein docking software ZDOCK 3.0.2, and 2000 complex structures were obtained. The RMSD values of these 2000 complexes were calculated as 3.0 $\AA$ A. All the structures in the largest cluster were sorted according to the free energy, and the conformation with the lowest binding free energy was selected as the conformation of PcRab7-VP28 complex (Figure 3). From Figure 3 we can easily found that, PcRab7 binds to VP28 through the second $\beta$-sheet, which is composed of residues L73-D86. In the previous research, Western blot and Dynabeads magnetic beads showed that the Rab7 protein of Penaeus chinensis (PcRab7) could bind to VP28 in the same way as the Rab7 protein of Penaeus monodon (PmRab7). Furthermore, Western blot and FIA were used to further

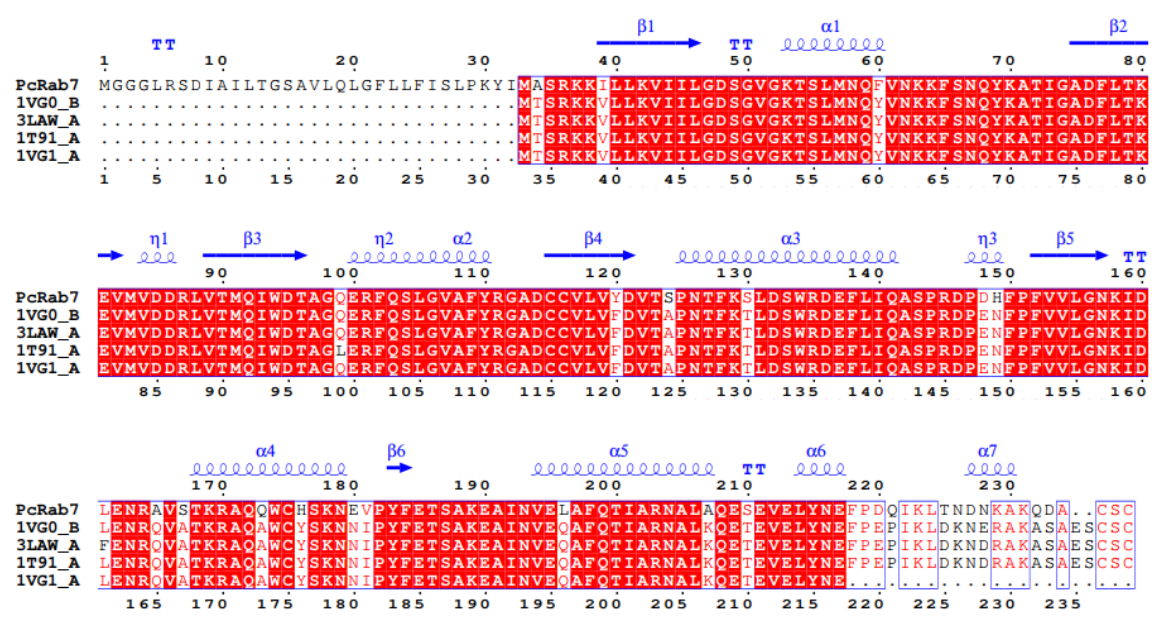

Figure 1. Multiple sequence alignment of PcRab7 with 1VG0, 3LAW, 1T91 and 1VG1. The blue spiral and arrow at the top represent the $\alpha$-helix and $\beta$-sheet. This figure was generated by Espript2.2. 

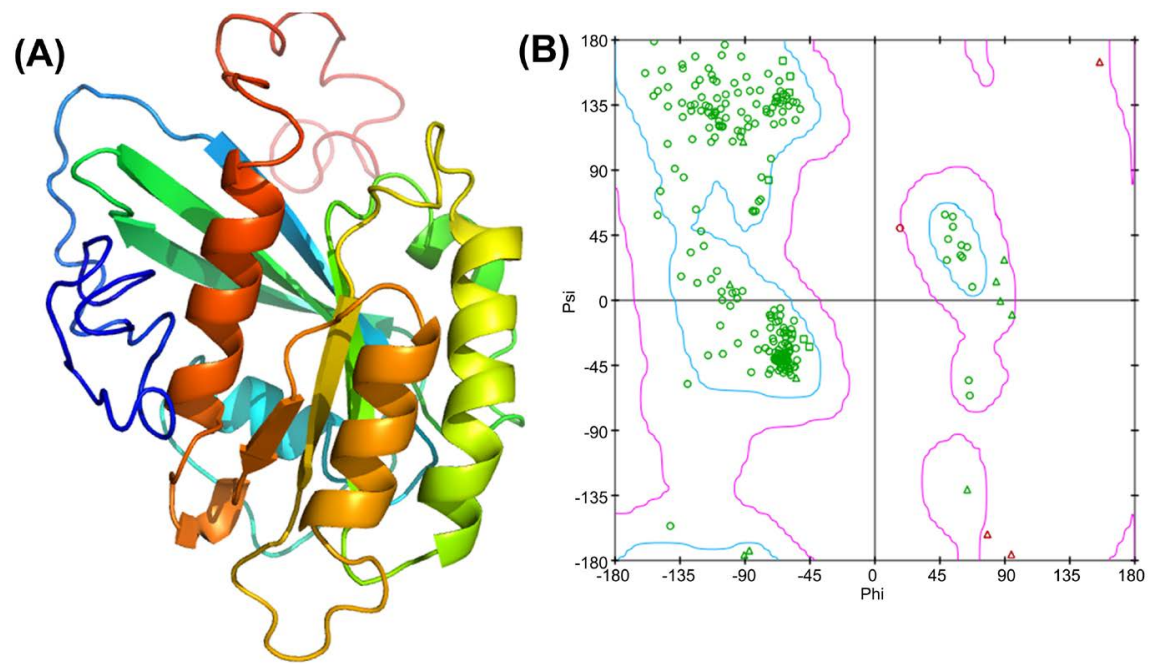

Figure 2. PcRab7 modelled structure and its rationality test. (A) The cartoon image of the tertiary structure of PcRab7 is generated by the molecular graphics software PyMOL and displayed in rainbow color (N-terminal is blue, C-terminal is red); (B) According to the Ramachandran plot test of PcRab7, the image was generated by DS4.2, in which the red triangle represents the residues in the unacceptable range.

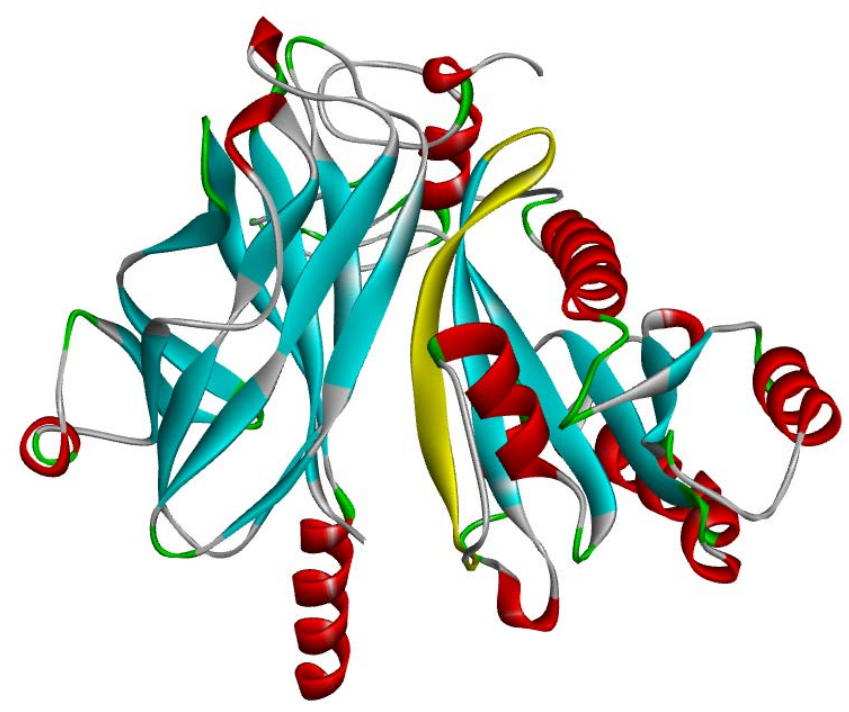

Figure 3. Complex of PcRab7 and VP28. VP28 on the left and PcRab7 on the right. Binding zone in PcRab7 was shown in yellow, $\alpha$-helix, $\beta$-sheets and loops were shown in red, blue and gray respectively.

analyze the specific action fragments of PcRab7 and VP28, and the preliminary conclusion was that the L73-T111 and P157-D192 (L41-T79 and P125-D160 in PmRab7) regions of PcRab7 had binding activity with VP28. Results of region prediction analysis based on docking study are consistent with those based on experiment. Through analysis of the interaction interface of the complex structure, we can see that E81, F77 and D76 of PcRab7 bind to VP28 by Pi-Pi and Pi-Alkey interaction (Figure 4). Therefore, residues E81, F77 and D76 were speculated to be the key site affecting the binding PcRab7 and VP28. 


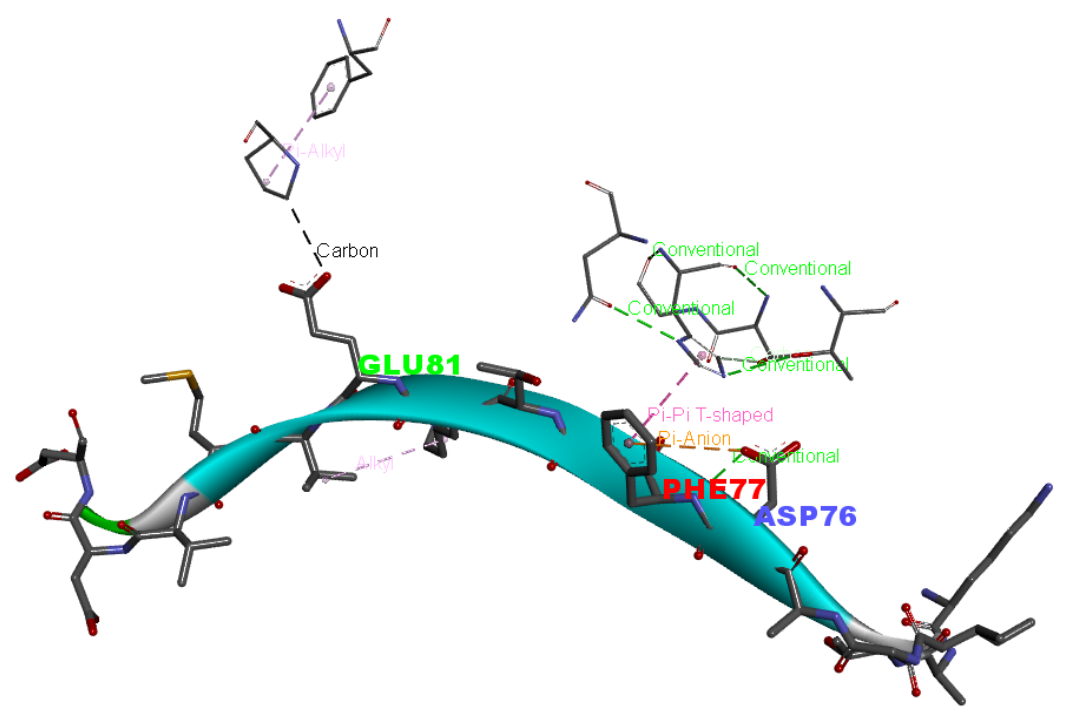

Figure 4. Binding sites of PcRab7 and VP28. Residues in PcRab7 were shown in cartoon stick and those in VP28 were shown in stick.

\section{Conclusion}

It is not difficult to find that the interaction mechanism of PcRab7 and VP28 is important and urgent in both theoretical research and application needs. To solve this problem, this research first modeled the tertiary structure of PcRab7 and then docked it to VP28 by employing the method of protein-protein docking. Furthermore, we determined the interaction interface is in the zone of L73-D86, which is consistent with the previous experimental results. Meanwhile the interaction sites of complex of PcRab7 and VP28 in PcRab7 are E81, F77 and D76. These results will contribute to a deep understanding of the infection mechanism of WSSV on the host, and provide a target and theoretical reference for further drug screening and design.

\section{Acknowledgements}

This work is supported by the Central Public-interest Scientific Institution Basal Research Fund, YSFRI, CAFS (NO. 20603022019023 and 20603022017006).

\section{Conflicts of Interest}

The authors declare no conflicts of interest regarding the publication of this paper.

\section{References}

[1] OIE (2003) Manual of Diagnostic Tests for Aquatic Animals, White Spot Disease. Office International des Epizooties, Paris, Chapter 4.1.2.

[2] Thitamadee, S., Prachumwat, A., Srisala, J., et al. (2016) Review of Current Disease Threats for Cultivated Penaeid Shrimp in Asia. Aquaculture, 452, 69-87. https://doi.org/10.1016/j.aquaculture.2015.10.028

[3] Rodriguez-Anaya, L.Z., Gonzalez-Galaviz, J.R., Casillas-Hernandez, R., et al. (2016) 
Draft Genome Sequence of White Spot Syndrome Virus Isolated from Cultured $\mathbf{L i}$ topenaeus vannamei in Mexico. Genome Announcement, 4, e01674-15. https://doi.org/10.1128/genomeA.01674-15

[4] Xie, X. and Yang, F. (2006) White Spot Syndrome Virus VP24 Interacts with VP28 and Is Involved in Virus Infection. Journal of General Virology, 87, 1903. https://doi.org/10.1099/vir.0.81570-0

[5] Sun, L., Su, Y., Zhao, Y., et al. (2016) Crystal Structure of Major Envelope Protein VP24 from White Spot Syndrome Virus. Scientific Reports, 6, Article No. 32309. https://doi.org/10.1038/srep32309

[6] Sritunyalucksana, K., Wannapapho, W., Lo, C.F. and Flegel, T.W. (2006) PmRab7 Is a VP28-Binding Protein Involved in White Spot Syndrome Virus Infection in Shrimp. Journal of Virology, 80, 10734-10742. https://doi.org/10.1128/JVI.00349-06

[7] Tang, X., Zhai, F., Sheng, X., et al. (2017) The Roles of $\beta$-Integrin of Chinese Shrimp (Fenneropenaeus chinensis) in WSSV Infection. International Journal of Molecular Sciences, 18, 1465. https://doi.org/10.3390/ijms18071465

[8] Krishnankutty Chandrika, S. and Thavarool Puthiyedathu, S. (2021) Challenges and Prospects of Viral Envelope Protein VP28-Based Control Strategies to Combat White Spot Syndrome Virus in Penaeid Shrimps: A Review. Reviews in Aquaculture, 13, 734-743. https://doi.org/10.1111/raq.12497

[9] Momtaz, F., Foysal, J., Rahman, M. and Fotedar, R. (2019) Design of Epitope Based Vaccine against Shrimp White Spot Syndrome Virus (WSSV) by Targeting the Envelope Proteins: An Immunoinformatic Approach. Turkish Journal of Fisheries and Aquatic Sciences, 19, 149-159. https://doi.org/10.4194/1303-2712-v19 207

[10] van Hulten, M.C.W., Goldbach, R.W. and Vlak, J.M. (2000) Three Functionally Diverged Major Structural Proteins of White Spot Syndrome Virus Evolved by Gene Duplication. Journal of General Virology, 81, 2525-2529.

https://doi.org/10.1099/0022-1317-81-10-2525

[11] Zhang, X., Huang, G., Xu, X. and Hew, C.L. (2002) Identification and Localization of a Prawn White Spot Syndrome Virus Gene That Encodes an Envelope Protein. Journal of General Virology, 83, 1069. https://doi.org/10.1099/0022-1317-83-5-1069

[12] Yi, G., Wang, Z., Qi, Y., et al. (2004) Vp28 of Shrimp White Spot Syndrome Virus Is Involved in the Attachment and Penetration into Shrimp Cells. Journal of Biochemistry \& Molecular Biology, 37, 726-734. https://doi.org/10.5483/BMBRep.2004.37.6.726

[13] Verma, A.K., Gupta, S., Singh, S.P. and Nagpure, N.S. (2017) An Update on Mechanism of Entry of White Spot Syndrome Virus into Shrimps. Fish \& Shellfish Immunology, 67, 141-146. https://doi.org/10.1016/j.fsi.2017.06.007

[14] van Hulten, M.C.W., Witteveldt, J., Snippe, M. and Vlak, J.M. (2001) White Spot Syndrome Virus Envelope Protein VP28 Is Involved in the Systemic Infection of Shrimp. Virology, 285, 228-233. https://doi.org/10.1006/viro.2001.0928

[15] Gouet, P., Robert, X. and Courcelle, E. (2003) ESPript/ENDscript: Extracting and Rendering Sequence and 3D Information from Atomic Structures of Proteins. Nucleic Acids Research, 31, 3320-3323. https://doi.org/10.1093/nar/gkg556

[16] Yang, J., Yan, R., Roy, A., et al. (2014) The I-TASSER Suite: Protein Structure and Function Prediction. Nature Methods, 12, 7-8. https://doi.org/10.1038/nmeth.3213

[17] Laskowski, R.A., MacArthur, M.W., Moss, D.S. and Thornton, J.M. (1993) PROCHECK: A Program to Check the Stereochemical Quality of Protein Structures. Journal of Applied Crystallography, 26, 283-291. 
https://doi.org/10.1107/S0021889892009944

[18] Pierce, B.G., Hourai, Y. and Weng, Z. (2011) Accelerating Protein Docking in ZDOCK Using an Advanced 3D Convolution Library. PLoS ONE, 6, e24657.

https://doi.org/10.1371/journal.pone.0024657 